\title{
Hyperbolic spoof plasmonic metasurfaces
}

\author{
Yihao Yang ${ }^{1,2,3,6}$, Liqiao Jing ${ }^{1,2,6}$, Lian Shen ${ }^{1,2}$, Zuojia Wang ${ }^{1,2}$, Bin Zheng ${ }^{1,2}$, Huaping Wang ${ }^{1,4}$, Erping $\mathrm{Li}^{2}$, \\ Nian-Hai Shen ${ }^{3}$, Thomas Koschny ${ }^{3}$, Costas M Soukoulis ${ }^{3,5}$ and Hongsheng Chen ${ }^{1,2}$
}

Hyperbolic metasurfaces have recently emerged as a new research frontier because of the unprecedented capabilities to manipulate surface plasmon polaritons (SPPs) and many potential applications. However, thus far, the existence of hyperbolic metasurfaces has neither been observed nor predicted at low frequencies because noble metals cannot support SPPs at longer wavelengths. Here, we propose and experimentally demonstrate spoof plasmonic metasurfaces with a hyperbolic dispersion, where the spoof SPPs propagate on complementary $\mathrm{H}$-shaped, perfectly conducting surfaces at low frequencies. Thus, non-divergent diffractions, negative refraction and dispersion-dependent spin-momentum locking are observed as the spoof SPPs travel over the hyperbolic spoof plasmonic metasurfaces (HSPMs). The HSPMs provide fundamental new platforms to explore the propagation and spin of spoof SPPs. They show great capabilities for designing advanced surface wave devices such as spatial multiplexers, focusing and imaging devices, planar hyperlenses, and dispersion-dependent directional couplers, at both microwave and terahertz frequencies. NPG Asia Materials (2017) 9, e428; doi:10.1038/am.2017.158; published online 25 August 2017

\section{INTRODUCTION}

Metamaterial is an artificial material with subwavelength unit cells that exhibit unusual properties that are never found in nature. ${ }^{1}$ Hyperbolic metamaterials, as some of the most unique metamaterials, have recently drawn significant attention from both physics and engineering communities because of their unconventional ability to manipulate light ${ }^{2}$ and due to their many potential applications, such as negative refraction, ${ }^{3}$ hyperlens, ${ }^{4}$ strong enhanced spontaneous emission, ${ }^{5,6}$ strong absorption ${ }^{7}$ and diverging local density of state. ${ }^{8}$ Because the three-dimensional hyperbolic metamaterials suffer from large volume, complexity of fabrication and considerable propagation losses, ${ }^{9}$ hyperbolic metasurfaces have been reported with the possibilities to circumvent these limitations due to the ultrathin nature of metasurfaces. ${ }^{10-16}$ The hyperbolic metasurfaces exhibit excellent compatibility with photonic integrated circuits and optoelectronic components and provide a flexible control of the transverse spin of the surface plasmon polariton (SPPs). ${ }^{10}$ The hyperbolic metasurfaces in practice may be implemented with plasmonic materials at high frequency. For example, at optical frequency, silver-air grating metasurfaces show a hyperbolic dispersion, ${ }^{11-12}$ which has been experimentally demonstrated very recently. ${ }^{13}$ While at terahertz and infrared frequencies, it has been theoretically predicted that uniform graphene nanostrips may provide a hyperbolic uniaxial conductivity. ${ }^{14}$
However, thus far, the existence of hyperbolic metasurfaces has neither been observed nor predicted at low frequencies because noble metals cannot support SPPs at longer wavelengths. Therefore, spoof SPPs or designer SPPs, which propagate on structured perfect electric conductors to mimic the optical properties of SPPs, such as dispersion behaviors and light confinement, ${ }^{17}$ have been recently proposed by Pendry et al. Unlike SPPs, ${ }^{18-21}$ which arise from the interaction between light and free electrons in the noble metals, spoof SPPs result from interactions between electromagnetic waves and spatial capacitances and inductances that are induced by the structured metal surfaces. Recent research on spoof SPPs has revealed their promising potential applications, such as in flexible waveguides, ${ }^{22}$ sensors, ${ }^{23}$ laser beams, ${ }^{24}$ energy concentration ${ }^{25}$ and integrated circuits. ${ }^{26}$ The concept that underlies spoof SPPs has also been extended to other physical systems, including acoustic waves, and has inspired novel devices in, for example, subwavelength imaging ${ }^{27}$ and collimation. ${ }^{28}$

Here, we propose and experimentally demonstrate plasmonic metasurfaces with a hyperbolic dispersion where the SPPs propagate on complementary $\mathrm{H}$-shaped, perfectly conducting surfaces at low frequencies. Thus, non-divergent diffractions, negative refraction and dispersion-dependent spin-momentum locking are observed as the spoof SPPs propagate over the hyperbolic spoof plasmonic metasurfaces (HSPMs). Compared with traditional bulky metamaterials, the HSPMs exhibit low propagation losses, are highly compatible with

\footnotetext{
${ }^{1}$ State Key Laboratory of Modern Optical Instrumentation, Zhejiang University, Hangzhou, China; ${ }^{2}$ The Electromagnetics Academy at Zhejiang University, College of Information Science \& Electronic Engineering, Zhejiang University, Hangzhou, China; ${ }^{3}$ Department of Physics and Astronomy and Ames Laboratory-U.S. DOE lowa State University, Ames, IA, USA; ${ }^{4}$ Institute of Marine Electronics Engineering, Zhejiang University, Hangzhou, China and ${ }^{5}$ Institute of Electronic Structure and Laser, FORTH, Heraklion, Greece

${ }^{6}$ These authors contributed equally to this work.

Correspondence: Professor H Wang, Institute of Marine Electronics Engineering, Ocean College, Zhejiang University, Hangzhou 310058, China.

E-mail: hpwang@zju.edu.cn

or Professor CM Soukoulis, A519 Zaffarano Hall, lowa State University, Ames, IA 5001, USA.

E-mail: soukoulis@ameslab.gov

or Professor H Chen, Zhejiang University, Room 221, Administration building, Yuquan Campus, Hangzhou 310058, China.

E-mail: hansomchen@zju.edu.cn

Received 29 March 2017; revised 17 June 2017; accepted 27 June 2017
} 

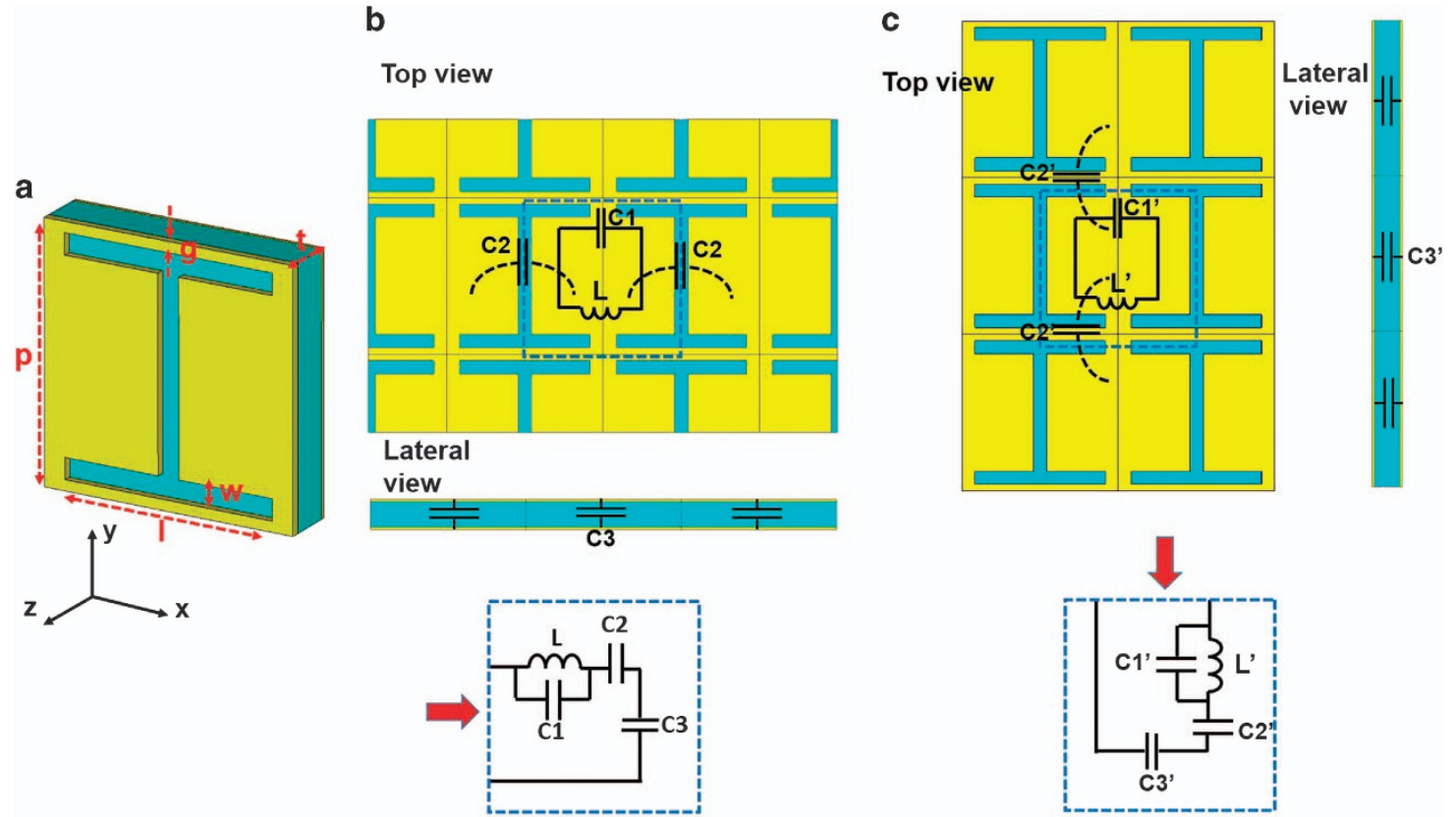

Figure 1 Unit cell of the HSPMs and its equivalent circuit model. (a) Unit cell of the HSPMs, where $p=6 \mathrm{~mm}, I=5 \mathrm{~mm}, w=0.5 \mathrm{~mm}, g=0.25 \mathrm{~mm}$, $t=1 \mathrm{~mm}$ and the thickness of the copper layer is $0.035 \mathrm{~mm}$. (b, c) Equivalent circuit models when electromagnetic waves propagate along the $x$ and $y$ directions, respectively. HSPM, hyperbolic spoof plasmonic metasurfaces.

integrated metamaterial circuits and may find potential applications in spatial multiplexers, focusing and imaging devices such as hyperlenses, dispersion-dependent directional couplers and photonic integrated circuits.

\section{MATERIALS AND METHODS}

The unit cell of the proposed HSPMs has a sandwich structure, as shown in Figure 1a, which consists of a copper ground layer, a substrate with a permittivity of 2.55, and a complementary $\mathrm{H}$-shaped layer. The electromagnetic properties of this unit cell depend on the geometries. In our example, $p=6 \mathrm{~mm}, l=5 \mathrm{~mm}$, $w=0.5 \mathrm{~mm}, g=0.25 \mathrm{~mm}$ and $t=1 \mathrm{~mm}$, and the thickness of the metal is $0.035 \mathrm{~mm}$. The impinging waves on the HSPMs induce surface currents that oscillate on the metal surfaces because of the special geometries. Clearly, due to the lack of $\mathrm{C}_{4}$ symmetry, this unit cell exhibits different responses when electromagnetic waves propagate along the $x$ and $y$ directions. To gain a better understanding of the underlying mechanism, we consider equivalent circuit models in which spoof SPPs propagate along the $x$ (Figure 1b) and $y$ directions (Figure 1c). Here, the spoof surface plasma frequency is $f_{x}=1 / \sqrt{L\left(C_{1} C_{2}+C_{3}\left(C_{1}+C_{2}\right) /\left(C_{3}+C_{2}\right)\right)}$, when the in-plane wave vector is along the $x$ direction, and $f_{y}=1 / \sqrt{L\left(C_{1}{ }^{\prime} C_{2}{ }^{\prime}+C_{3}{ }^{\prime}\left(C_{1}{ }^{\prime}+C_{2}{ }^{\prime}\right) /\left(C_{3}{ }^{\prime}+C_{2}{ }^{\prime}\right)\right)}$, when the wave vector is along the $y$ direction, which mimics the surface plasmon frequencies of noble metals at optical frequencies. $C_{1}\left(C_{1}{ }^{\prime}\right), C_{2}\left(C_{2}{ }^{\prime}\right), C_{3}\left(C_{3}{ }^{\prime}\right)$ and $L(L$ ') are the capacitance inside the unit cell, the mutual capacitance between neighbor unit cells, the capacitance between two metal layers and the inductance inside the unit cell, respectively. When $f_{0}<f_{x}<f_{y}$ ( $f_{0}$ is the operational frequency), the spoof SPPs propagate along both orthogonal directions, and the equal-frequency contours (EFCs) are elliptical. Alternatively, when $f_{x}<f_{0}<f_{y}$, only the spoof SPPs propagating along the $y$ direction exist. By altering the geometries appropriately, one can tailor the EFCs to be hyperbolic. In addition, an effective surface conductivity model can also be used to characterize the present metasurface in a certain frequency band $^{10,14,16,29}$ (see the Supplementary Information, Supplementary Figure S2).

We numerically calculate the HSPM dispersions by employing the Eigenvalue module of the commercial Computer Simulation Technology (CST) Microwave Studio software (CST China Ltd., Shanghai, China). The electric field distributions for electromagnetic waves propagating along the $x$ and $y$ directions are shown in Figures 2a and b, respectively. The different field distributions indicate that the HSPMs manipulate the spoof SPPs along the $x$ and $y$ directions in different ways. Furthermore, the electric field distributions show that the gap- induced capacitances play important roles in the equivalent circuit models. The first-band EFCs of the metasurface unit cell in the first Brillouin zone are shown in Figure $2 \mathrm{c}$ and reflect a transition in the topology from a closed elliptical curve (below $6.0 \mathrm{GHz}$ ) to an open, extremely anisotropic curve, to a flat curve (from 9.0 to $10.0 \mathrm{GHz}$ ), and finally, to an open hyperbolic curve (from 10.0 to $11.0 \mathrm{GHz})$. By controlling the geometry-induced capacitances and inductances, the dispersions of the metasurface can be tailored to manipulate how the spoof SPPs propagate. Even without the bottom ground layer and mediated dielectric, a single layer of conducting surface with the proposed pattern can also exhibit a topological transition of EFCs (see Supplementary Figure S1). However, considering the experimental convenience of exciting spoof SPPs, we still apply a ground metal layer in both simulations and experiments. This additional ground layer allows the spoof SPPs to be controlled and excited more freely, as shown by the directional coupler designed at the end of the paper.

\section{RESULTS}

In the following, we demonstrate the electromagnetic properties of the proposed metasurface, including the topological transition of EFCs in the wave vector space, frequency-dependent spatial localization, nondiffraction propagation, negative refraction and dispersion-dependent spin-momentum locking of spoof SPPs. The spoof SPP modes are excited by an electric dipole between the two metal layers of the metasurface. Here, the HSPMs have dimensions of $300 \mathrm{~mm}$ by $384 \mathrm{~mm}$, and the simulations are performed in the transient module of CST Microwave Studio. Figure 3 presents distributions of the $z$-component of an electric field positioned $5.6 \mathrm{~mm}$ above the metasurface and the corresponding EFCs at 6.0, 8.75, 9.25, 9.75, 10.25 and $10.75 \mathrm{GHz}$. Intriguingly, the wavefronts of the spoof SPPs in the $x y$ plane gradually change from convex to flat and then to concave. At $6.0 \mathrm{GHz}$, the corresponding EFC is a closed ellipse, and the spoof SPPs can propagate in any direction in the $x y$ plane (Figure 3a). When the frequency increases to $8.75 \mathrm{GHz}$, the EFCs become extremely anisotropic. Because the group velocity vectors of the spoof SPPs should be perpendicular to the EFCs, the spoof SPPs are guided and split into two beams because of the EFCs' special shapes. The propagation directions of the spoof SPPs are frequency dependent (Figures $3 b$ and c). This 
a

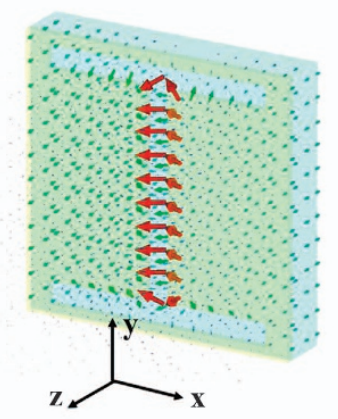

b

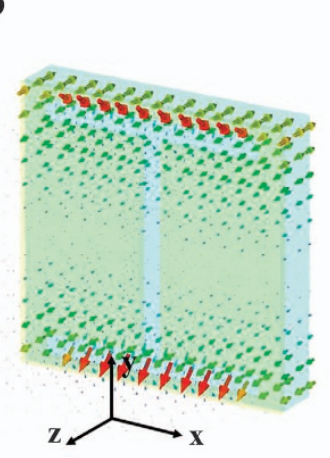

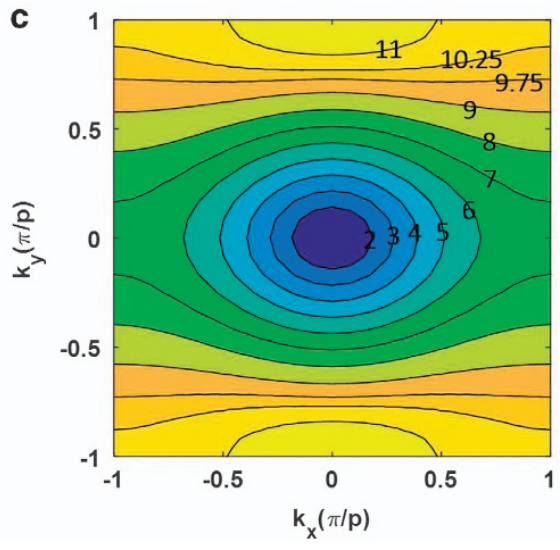

Figure 2 Dispersion of the HSPMs. (a, b) Electric field distributions when electromagnetic waves propagate along the $x$ and $y$ directions, respectively. (c) First-band equal-frequency contours in the first Brillouin zone. The frequency values are presented in gigahertz (GHz). HSPM, hyperbolic spoof plasmonic metasurfaces.
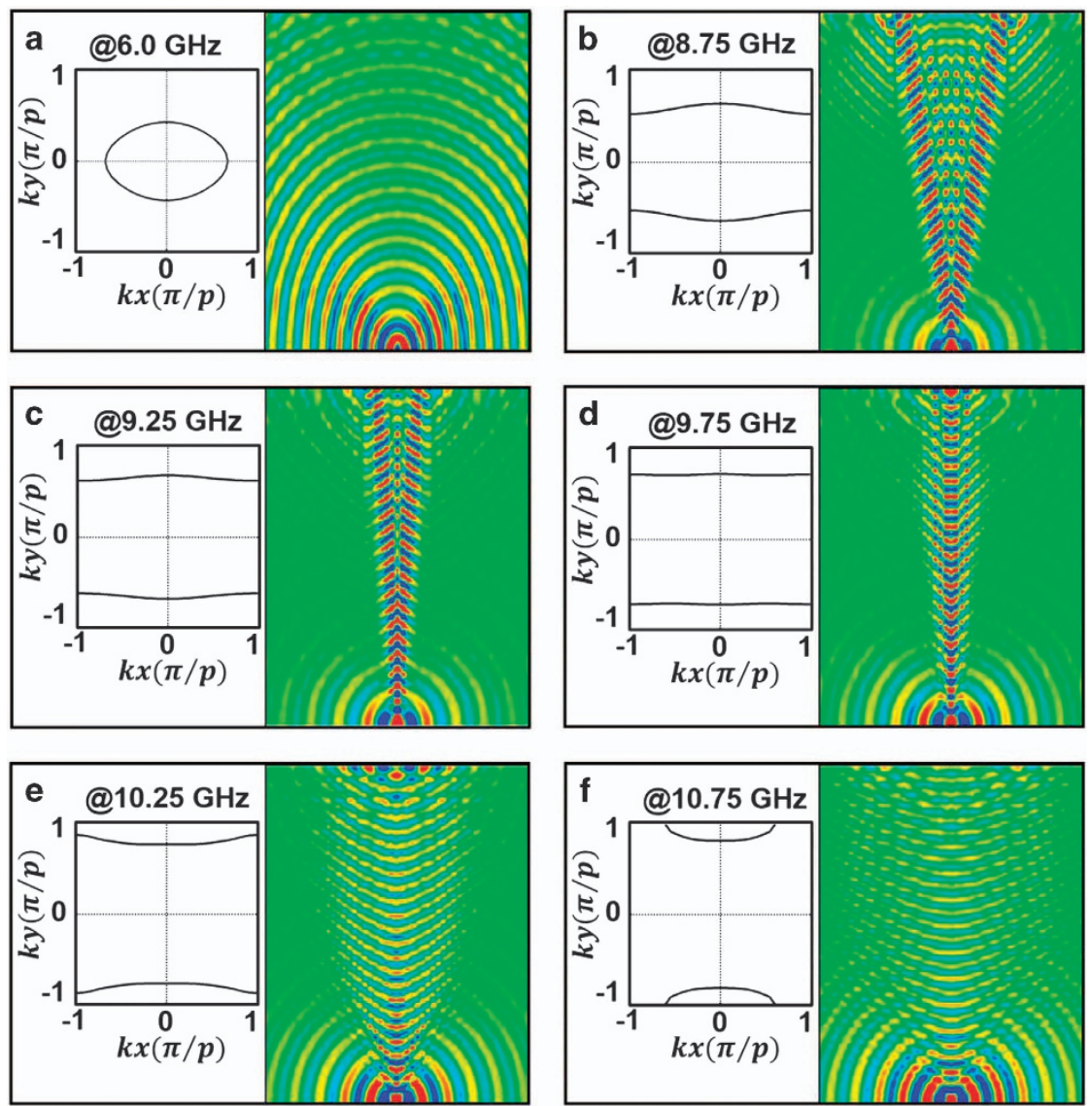

Figure 3 Simulated field distributions and EFCs. (a, f) EFCs and simulated Ez field distributions in the $x y$ plane $5.6 \mathrm{~mm}$ above the metasurfaces at: (a) $6.0 \mathrm{GHz}$, (b) $8.75 \mathrm{GHz}$, (c) $9.25 \mathrm{GHz}$, (d) $9.75 \mathrm{GHz}$, (e) $10.25 \mathrm{GHz}$ and (f) $10.75 \mathrm{GHz}$. EFC, equal-frequency contour.

phenomenon can indeed be applied to design spatial multiplexers. At the transition point near $9.75 \mathrm{GHz}$, the spoof SPPs propagate in a selfcollimating manner because of the flat dispersion. ${ }^{30}$ This self-collimation phenomenon, which also exists in photonic crystals, may have potential applications in integrated surface wave circuit systems and hyperlenses ${ }^{11}$ (Figure 3d). From 9.75 to $10.75 \mathrm{GHz}$, the spoof SPPs propagate in a convergent manner because the EFCS are hyperbolic (Figures $3 e$ and f).

The topological transition of EFCs over the metasurfaces is also experimentally demonstrated. In this work, the complementary
$\mathrm{H}$-shaped resonator structures are printed on a substrate: a $1-\mathrm{mm}$ Teflon woven glass fabric copper-clad laminate with a permittivity of 2.55 and $\tan (\delta)<0.001$ at $10.0 \mathrm{GHz}$ (Supplementary Figure S3h). We use an electric dipole between the two metal layers to excite the spoof SPPs, which is equivalent to the source setting in the simulations. Therefore, the measured field distributions exactly match the simulated counterparts (Figures $4 \mathrm{a}-\mathrm{h}$ ). To obtain the surface current distributions, we use an electric dipole antenna placed on the metasurface to detect the surface current, point by point, with the 

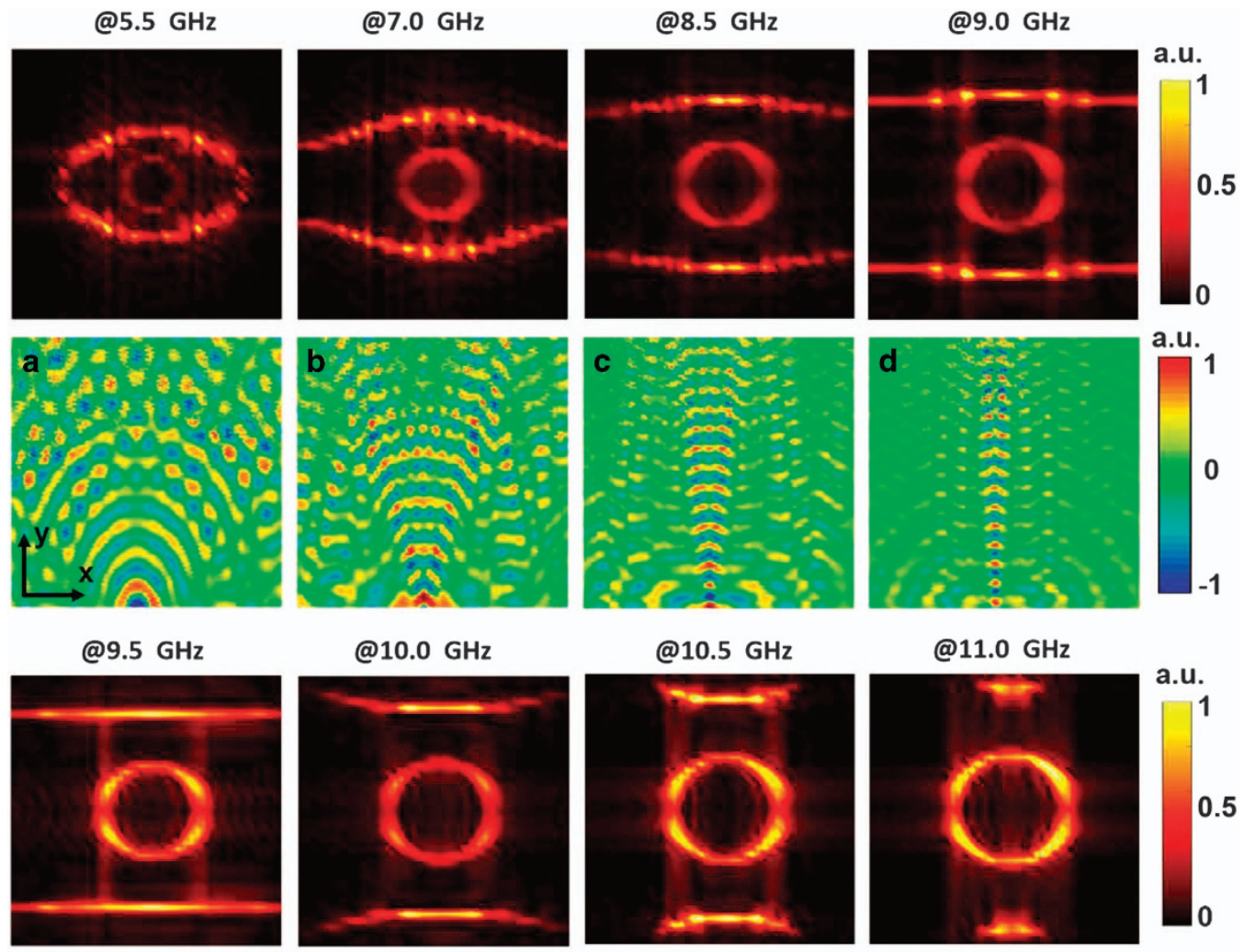

a.u.
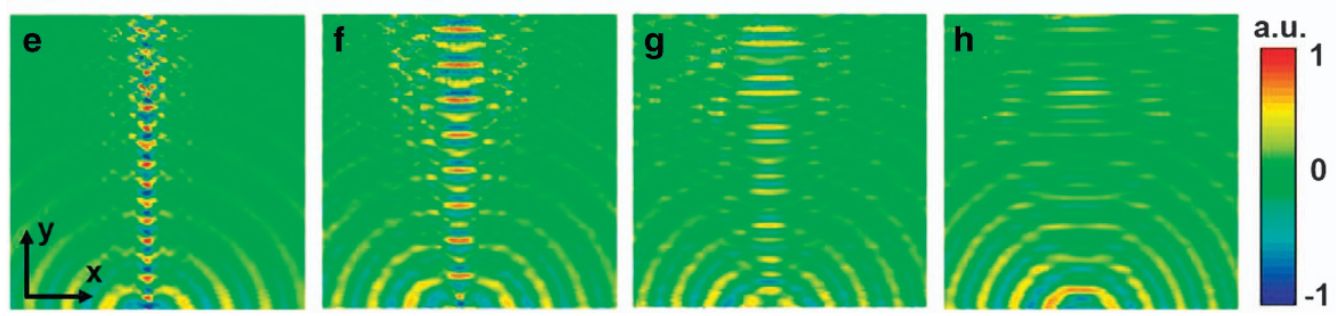

Figure 4 Measured field distributions and EFCs. Surface current distributions measured on the metasurfaces and the corresponding EFCs in the first Brillouin zone at: (a) $5.5 \mathrm{GHz}$, (b) $7.0 \mathrm{GHz}$, (c) $8.5 \mathrm{GHz}$, (d) $9.0 \mathrm{GHz}$, (e) $9.5 \mathrm{GHz}$, (f) $10.0 \mathrm{GHz}$, (g) $10.5 \mathrm{GHz}$ and (h) $11.0 \mathrm{GHz}$. EFC, equal-frequency contour.

aid of a platform that can be controlled in three dimensions. The measured region is $252 \mathrm{~mm}$ by $252 \mathrm{~mm}$, and the resolution is $2 \mathrm{~mm}$ by $2 \mathrm{~mm}$. We apply a spatial Fourier transform to obtain the EFCs in the k-vector space, as shown in Figure 3. Note that the $z$-oriented electric field distribution is also measured in the $x y$ plane $1 \mathrm{~mm}$ above the HSPMs, the results of which are shown in Supplementary Figure S3a-g. Both the EFCs and surface current distributions clearly exhibit the transition of EFCs from a closed elliptical line to a flat one and, finally, to a hyperbolic one, with the wave-front transiting from convex to flat and eventually, to concave. The circles at the centers of the EFCs in Figure 4 correspond to the dispersion of air. One may notice that the dispersion of the HSPMs is outside that of the air because the designer SPPs carry larger momentum than that in the air. At the transition point $(9.5 \mathrm{GHz})$, the spoof SPPs propagate in a nondiffracting manner, behaving analogously to spatial solitons in nonlinear optics, but purely based on a linear optical system. ${ }^{31}$

If an appropriate background metasurface was chosen (Figures 5b and c), the negative refraction of spoof SPPs will occur at the interface between the HSPMs and the background metasurface (Figure 5a). The EFCs of the background metasurface and the HSPMs at $10.6 \mathrm{GHz}$ are shown in Figure 5d. All incoming wave vectors at this frequency are included within the EFCs, which results in an all-angle negative refraction.
Thus, they can be applied to spoof SPP focusing and imaging. ${ }^{32}$ In our experiments, we chose air as the background because when the spoof SPPs scatter into air, their energy becomes focused (Figure 5e). Therefore, the HSPMs function as ultrathin planar imaging devices, which may lead to some valuable applications, especially for terahertz and far-infrared frequencies.

The HSPMs can control not only the propagation of spoof SPPs but also their transverse spins. Very recently, Bliokh et al. ${ }^{33}$ theoretically demonstrated that the free-space light exhibits an intrinsic quantum spin Hall effect and that the surface modes, such as SPPs and spoof SPPs, exhibit strong spin-momentum locking. Therefore, the surface waves that propagate left and right will carry transverse spin with opposite directions at the metal-dielectric interfaces; this phenomenon is the so-called spin Hall effect of light and has been demonstrated in several experiments. ${ }^{34-35}$ In our case, we place an electric dipole on the metasurface and excite spoof SPPs with a different transverse spin that propagates left and right. By tailoring the dispersion of the metasurface, the transverse spin of the spoof SPPs can be engineered. When the EFCs are elliptical, the spoof SPPs that propagate in the left and right directions exhibit right-handed $\left(E_{x}-i E_{z}\right)$ and left-handed $\left(E_{x}+i E_{z}\right)$ spin relative to the normal incident case (Figures 6a and d). When the EFCs are flat, the spoof SPPs propagate only in the $y$ 


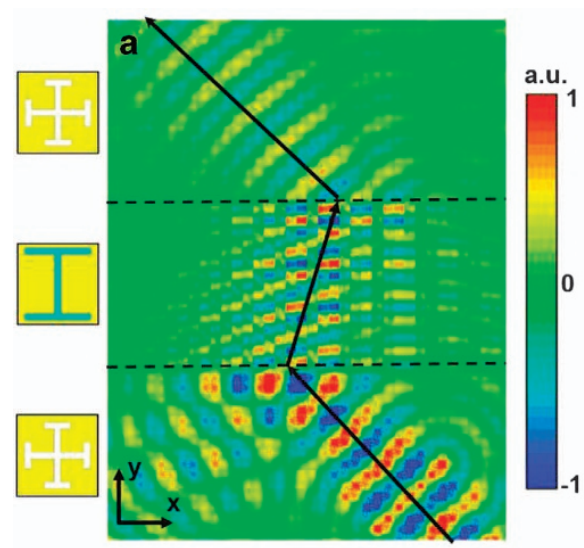

b
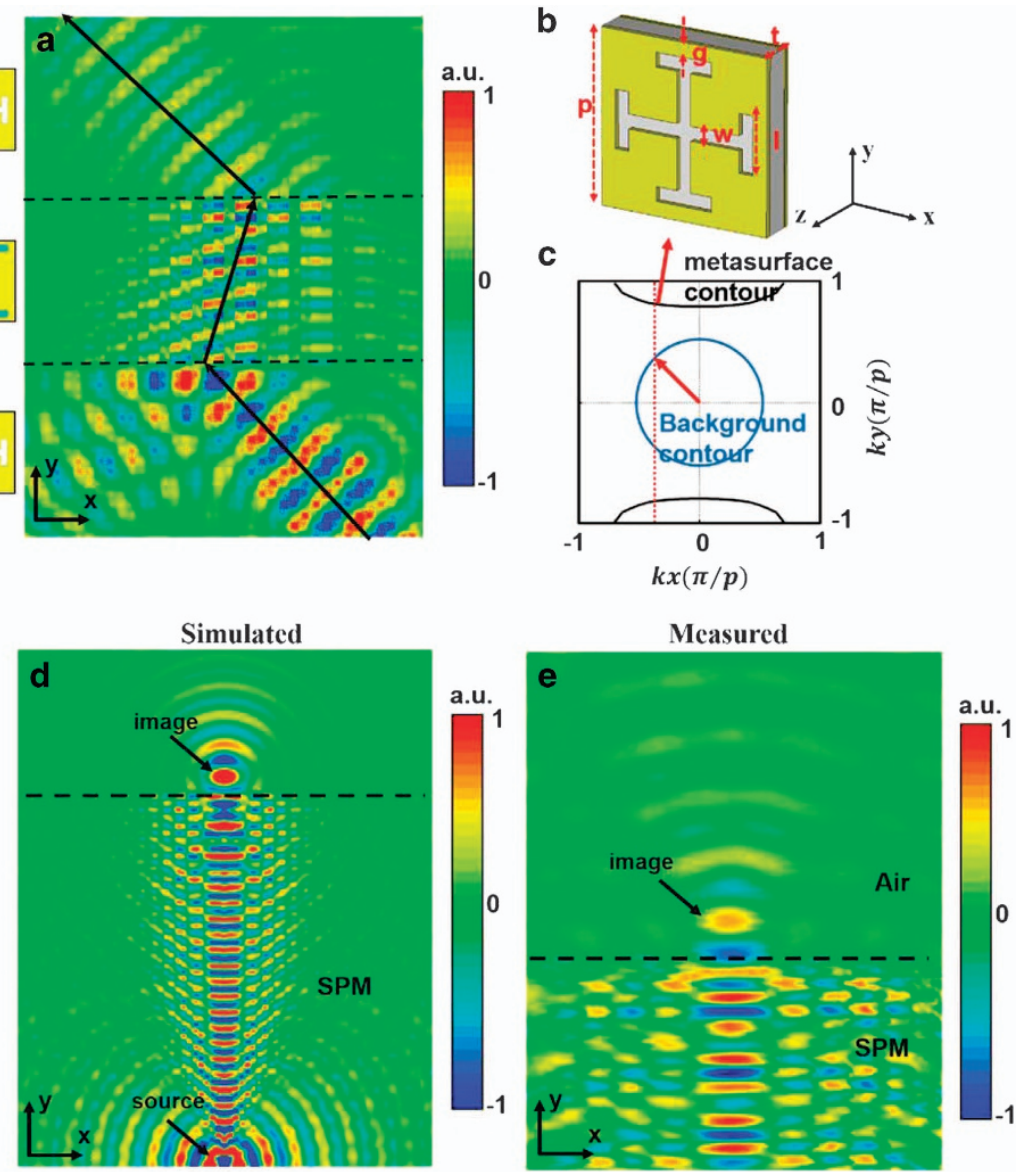

Figure 5 Negative refraction of spoof SPPs and imaging devices. (a) Ez field distribution in the $x y$ plane $0.5 \mathrm{~mm}$ below the patterned metal surface. When spoof SPPs in a background metasurface are incident to an HSPM, negative refraction phenomena will occur at the interface at $10.6 \mathrm{GHz}$. (b) Geometrical parameters of the background metasurface; $p=5 \mathrm{~mm}, g=0.5 \mathrm{~mm}, w=0.5 \mathrm{~mm}, l=2 \mathrm{~mm}$ and $t=1 \mathrm{~mm}$, and the material between the two metal layers is air. (c) EFC at $10.6 \mathrm{GHz}$. The blue and black curves are the EFCs of the background metasurface and HSPMs, respectively. The red arrows represent the propagation direction of the spoof SPPs. (d, e) Simulated and measured Ez fields in the $x y$ plane $1 \mathrm{~mm}$ above the HSPM as the spoof SPPs propagate into the surrounding medium at $10.2 \mathrm{GHz}$. The background medium is air, and the spoof SPPs scattering into the air are focused. EFC, equal-frequency contour; HSPM, hyperbolic spoof plasmonic metasurfaces; SPP, surface plasmon polariton.

direction, and the right-handed and left-handed spin in the $x z$-plane cancel each other out ${ }^{36}$ (Figures $6 \mathrm{~b}$ and e). When the EFCs are hyperbolic, the spoof SPPs carry an opposite transverse spin (Figures $6 \mathrm{c}$ and $\mathrm{f}$ ) relative to the normal cases. Moreover, because of the angle-dependent local density of electromagnetic states, the propagation of spoof SPPs is strongly directional (Figures 6a and d). This phenomenon arises physically from the spin-orbit coupling and can be explained by the dispersion of the HSPMs (Figure $6 \mathrm{~g}$ ). The spoof SPP modes with right- and left-handed transverse spin are in the left and right wave vector space, respectively, and their propagation directions should be perpendicular to the EFCs.

Based on the dispersion-dependent spin Hall effect, we design a coupler to launch divergent, soliton-like or convergent spoof SPPs directionally over the HSPMs, which are controlled by the polarization of the incident electromagnetic waves. As shown in Figure 7a, the coupler is composed of two columns of subwavelength narrow apertures in the bottom metal film of the HSPM. If the coupler is well-designed, destructive interferences will occur on one side of the columns, and constructive interference will occur on the other side simultaneously, which results in the unidirectional launching of the surface waves. ${ }^{34,37}$ Here, by choosing $w=0.5 \mathrm{~mm}$, $l=3 \mathrm{~mm}, p=4 \mathrm{~mm}$ and $g=\lambda_{0} / 4=4.1 \mathrm{~mm}$, where $\lambda_{0}$ is the wavelength of the spoof SPPs at $9.75 \mathrm{GHz}$, in our simulations, we successfully excite soliton-like spoof SPPs over the HSPMs in a unidirectional (Figures 7b and c) or bi-directional manner (Figure 7d) at $9.75 \mathrm{GHz}$. This phenomenon is also demonstrated experimentally, and both unidirectional (Figures $7 \mathrm{e}$ and $\mathrm{f}$ ) and bi-directional (Figure $7 \mathrm{~g}$ ) launching of the soliton-like spoof SPPs is observed. The results can be applied to directionally launch spoof SPPs and detect the polarization of electromagnetic waves. The wavefronts of spoof SPPs with arbitrary shapes can be tailored by further engineering the apertures. ${ }^{38}$ Thus, the HSPMs may serve as a platform for exploring the properties of the transverse spin of spoof SPPs with different dispersions.

\section{DISCUSSION}

In this work, we propose and experimentally characterize ultrathin HSPMs at low frequencies. The HSPMs provide a fundamental new platform to explore the propagation and spin of the spoof SPPs. We experimentally observe numerous phenomena over the HSPMs, including frequency-dependent spatial localization, non-diffraction propagation, negative refraction and the dispersion-dependent spin Hall effect. Compared with three-dimensional metamaterials, the twodimensional HSPMs show the unconventional property of controlling spoof SPPs without suffering large losses and have advantages regarding much more convenient fabrication and superior compatibility with 


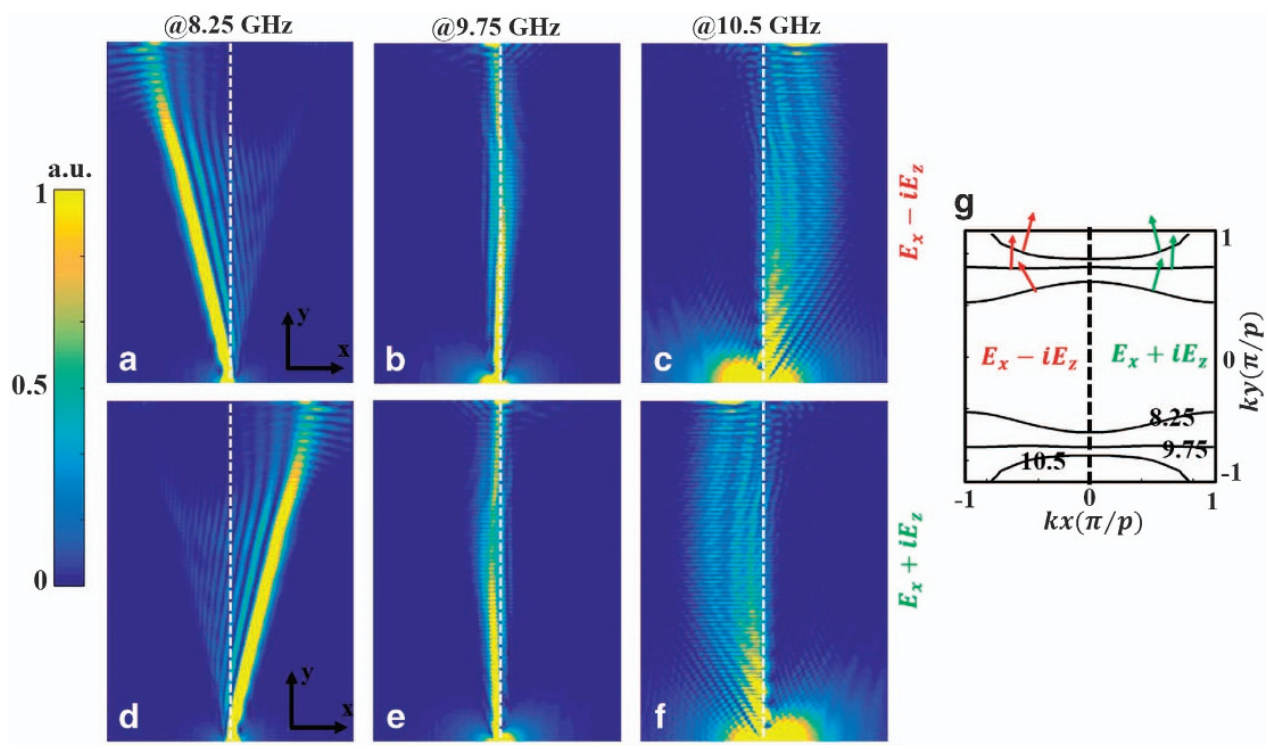

Figure 6 Dispersion-dependent spin-momentum locking of spoof SPPs. (a-c) Electric field intensity distributions of the right-handed spin $\left(E_{x}-i E_{z}\right)$ spoof SPPs in the $x y$ plane $5.6 \mathrm{~mm}$ above the HSPMs at $8.25,9.75$ and $10.5 \mathrm{GHz}$, respectively. (d-f) Electric field intensity distributions of the left-handed spin $\left(E_{x}+i E_{z}\right)$ spoof SPPs propagating in the $x y$ plane $5.6 \mathrm{~mm}$ above the HSPMs at $8.25,9.75$ and $10.5 \mathrm{GHz}$, respectively. (g) EFCs at $8.25,9.75 \mathrm{and} 10.5 \mathrm{GHz}$. Red and green arrows represent the propagation directions of the spoof SPPs with right-handed and left-handed spin, respectively. EFC, equal-frequency contour; HSPM, hyperbolic spoof plasmonic metasurfaces; SPP, surface plasmon polariton.

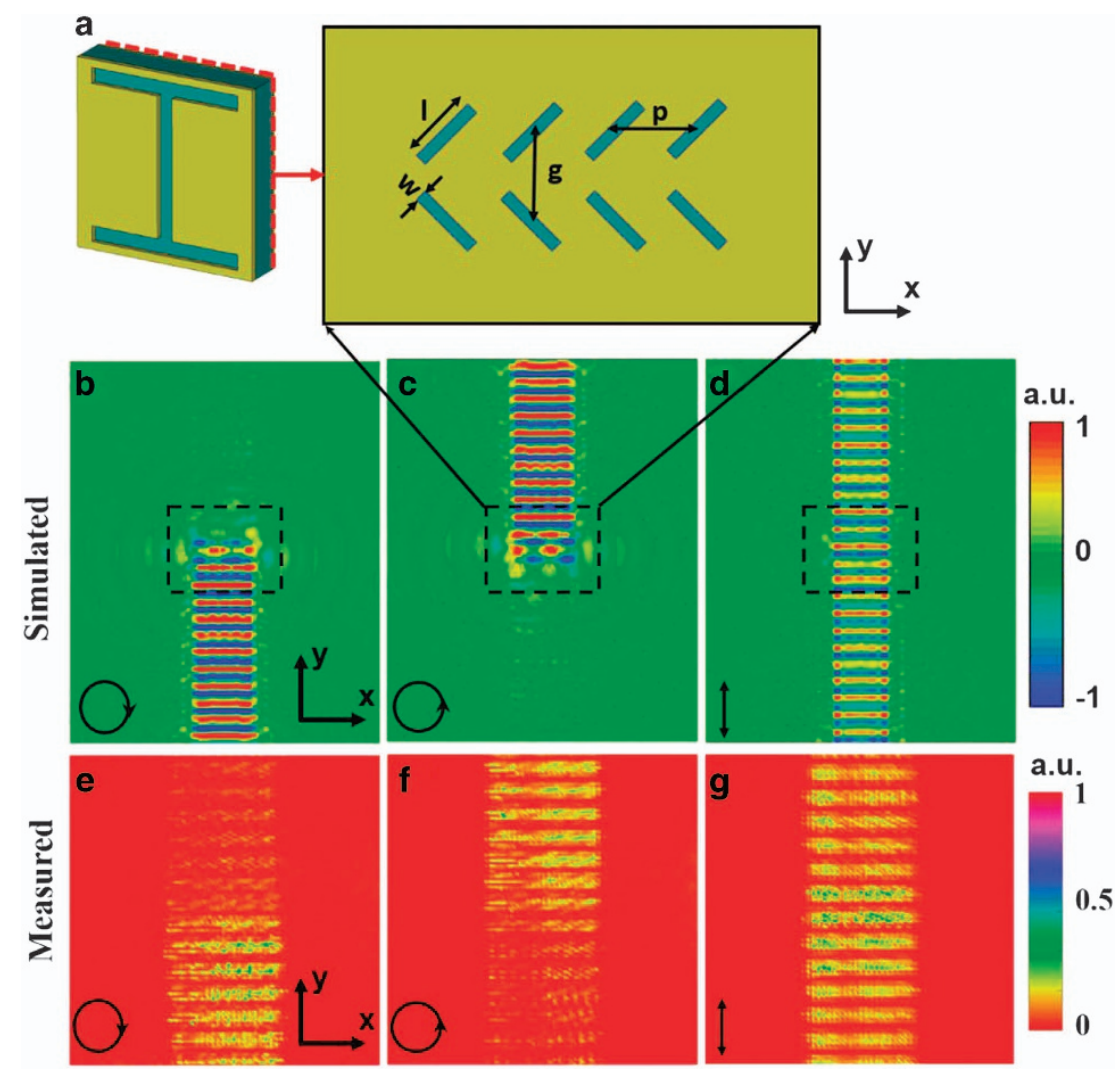

Figure 7 Dispersion-dependent directional coupler. (a) Schematic of the directional coupler composed of two columns of subwavelength narrow apertures in the bottom metal film of the HSPMs, where $w=0.5 \mathrm{~mm}, I=3 \mathrm{~mm}, p=4 \mathrm{~mm}$ and $g=4.1 \mathrm{~mm}$. (b-d) Simulated $E z$ field distributions of soliton-like spoof SPPs in the $x y$ plane $5 \mathrm{~mm}$ above the HSPM when a plane electromagnetic wave with right circular (b), left circular (c) or linear polarization (d) is incident onto the HSPMs from the bottom layer side at $9.75 \mathrm{GHz}$. (e-g) Measured surface current density of soliton-like spoof SPPs measured over the HSPM when plane waves with right circular (e), left circular (f) and linear polarization (g) are incident onto the HSPMs from the bottom layer side at $9.8 \mathrm{GHz}$. HSPM, hyperbolic spoof plasmonic metasurfaces; SPP, surface plasmon polariton. 
photonic integrated circuits. We believe that the presented metasurface will be promising, for example, in broad applications in spatial multiplexers, focusing and imaging devices, planar hyperlenses, dispersion-dependent directional couplers, and photonic integrated circuits. With the introduction of actively controllable components, ${ }^{26}$ one can dynamically tailor the dispersions of the HSPMs and realize the tunable characteristics of spoof SPPs including their propagation and spin. Furthermore, the HSPMs will also be beneficial for building two-dimensional transformation-optics-based devices. Finally, because the HSPMs arise from structures, their underlying concept may be extended to other physical systems, such as acoustic waves, and inspire the development of novel acoustic surface wave devices.

\section{CONFLICT OF INTEREST}

The authors declare no conflict of interest.

\section{ACKNOWLEDGEMENTS}

The work at Zhejiang University was sponsored by the National Natural Science Foundation of China under Grants No. 61625502, No. 61574127, and No. 61601408, the ZJNSF under Grant No. LY17F010008, the Top-Notch Young Talents Program of China, the Fundamental Research Funds for the Central Universities under Grant No. 2017XZZX008-06, and the Innovation Joint Research Center for Cyber-Physical-Society System. The work at the Ames Laboratory was partially supported by the U.S. Department of Energy, Office of Basic Energy Science, Division of Materials Sciences and Engineering (Ames Laboratory is operated for the U.S. Department of Energy by Iowa State University under Contract No. DE-AC02-07CH11358). The European Research Council supported the work at FORTH via ERC Advanced Grant No. 320081 (PHOTOMETA). The authors thank Zheping Shao for the help with the measurement, and Xiao Lin for fruitful discussions.

\section{PUBLISHER'S NOTE}

Springer Nature remains neutral with regard to jurisdictional claims in published maps and institutional affiliations.

1 Wegener, M. Materials science. Metamaterials beyond optics. Science $\mathbf{3 4 2}$, 939-940 (2013).

2 Poddubny, A., Iorsh, I., Belov, P. \& Kivshar, Y. Hyperbolic metamaterials. Nat. Photon. 7, 948-957 (2013).

3 Fang, A., Koschny, T. \& Soukoulis, C. M. Optical anisotropic metamaterials: negative refraction and focusing. Phys. Rev. B 79, 245127 (2009).

4 Fang, N., Lee, H., Sun, C. \& Zhang, X. Sub-diffraction-limited optical imaging with a silver superlens. Science 308, 534-537 (2005).

5 Poddubny, A. N., Belov, P. A. \& Kivshar, Y. S. Spontaneous radiation of a finite-size dipole emitter in hyperbolic media. Phys. Rev. A 84, 023807 (2011).

6 Lu, D., Kan, J. J., Fullerton, E. E. \& Liu, Z. Enhancing spontaneous emission rates of molecules using nanopatterned multilayer hyperbolic metamaterials. Nat. Nanotech. 9, 48-53 (2014).

7 Tumkur, T., Gu, L., Kitur, J., Narimanov, E. E. \& Noginov, M. Control of absorption with hyperbolic metamaterials. App. Phys. Lett. 100, 161103 (2012).

8 Krishnamoorthy, H. N., Jacob, Z., Narimanov, E., Kretzschmar, I. \& Menon, V. M. Topological transitions in metamaterials. Science 336, 205-209 (2012).

9 Shalaev, V. M. Optical negative-index metamaterials. Nat. photon. 1, 41-48 (2007).

10 Yermakov, Y., Ovcharenko, A. I., Bogdanov, A. A., Iorsh, I. V., Bliokh, K. Y. \& Kivshar, Y. S. Spin control of light with hyperbolic metasurfaces. Phys. Rev. B 94, 075446 (2016).

11 Kildishev, A. V., Boltasseva, A. \& Shalaev, V. M. Planar photonics with metasurfaces Science 339, 1232009 (2013).

12 Liu, Y. \& Zhang, X. Metasurfaces for manipulating surface plasmons. App. Phys. Lett. 103, 141101 (2013).

13 High, A. A., Devlin, R. C., Dibos, A., Polking, M., Wild, D. S., Perczel, J., Leon, N. P., Lukin, M. D. \& Park, H. Visible-frequency hyperbolic metasurface. Nature 522, 192-196 (2015),

14 Gomez-Diaz, J. S., Tymchenko, M. \& Alu, A. Hyperbolic plasmons and topological transitions over uniaxial metasurfaces. Phys. Rev. Lett. 114, 233901 (2015).
15 Yang, Y., Jing, L., Zheng, B., Hao, R., Yin, W., Li, E., Soukoulis, C. M. \& Chen, H. Fullpolarization 3D metasurface cloak with preserved amplitude and phase. Adv. Mater. 28, 6866-6871 (2016)

16 Yermakov, O. Y., Ovcharenko, A. I., Song, M., Bogdanov, A. A., Iorsh, I. V. \& Kivshar, Y. S. Hybrid waves localized at hyperbolic metasurfaces. Phys. Rev. B 91, 235423 (2015).

17 Pendry, J. B., Martin-Moreno, L. \& Garcia-Vidal, F. J. Mimicking surface plasmons with structured surfaces. Science 305, 847-848 (2004).

18 Li, R., Zheng, B., Lin, X., Hao, R., Lin, S., Yin, W., Li, E. \& Chen, H. Design of ultracompact graphene-based superscatterers. IEEE J. Sel. Top. Quantum Electron. 23, 1-8 (2017).

19 Lin, X., Kaminer, I., Shi, X., Gao, F., Yang, Z., Gao, Z., Buljan, H., Joannopoulos, J. D., Soljačić, M. \& Chen, H. Splashing transients of 2D plasmons launched by swift electrons. Sci. Adv. 3, e1601192 (2017).

20 Li, R., Imran, M., Lin, X., Wang, H., Xu, Z. \& Chen, H. Hybrid airy plasmons with dynamically steerable trajectories. Nanoscale 9, 1449-1456 (2017).

21 Barnes, W. L., Dereux, A. \& Ebbesen, T. W. Surface plasmon subwavelength optics. Nature 424, 824-830 (2003).

22 Shen, X., Cui, T. J., Martin-Cano, D. \& Garcia-Vidal, F. J. Conformal surface plasmons propagating on ultrathin and flexible films. Proc. Natl Acad. Sci. USA 110, 40-45 (2013).

23 Shen, X. \& Cui, T. J. Ultrathin plasmonic metamaterial for spoof localized surface plasmons. Laser Photon. Rev. 8, 137-145 (2014).

24 Yu, N., Wang, Q. J., Kats, M. A., Fan, J. A., Khanna, S. P., Li, L., Davies, A. G., Linfield, E. H. \& Capasso, F. Designer spoof surface plasmon structures collimate terahertz laser beams. Nat. Mater. 9, 730-735 (2010).

25 Maier, S. A., Andrews, S. R., Martin-Moreno, L. \& Garcia-Vidal, F. J. Terahertz surface plasmon-polariton propagation and focusing on periodically corrugated metal wires. Phys. Rev. Lett. 97, 176805 (2006).

26 Zhang, H. C., Liu, S., Shen, X., Chen, L. H., Li, L. \& Cui, T. J. Broadband amplification of spoof surface plasmon polaritons at microwave frequencies. Laser Photon. Rev. 9, 83-90 (2015).

27 Zhu, J., Christensen, J., Jung, J., Martin-Moreno, L., Yin, X., Fok, L., Zhang, X. \& Garcia-Vidal, F. J. A holey-structured metamaterial for acoustic deep-subwavelength imaging. Nat. Phys. 7, 52-55 (2010).

28 Christensen, J., Fernandez-Dominguez, A. I., de Leon-Perez, F., Martin-Moreno, L. \& Garcia-Vidal, F. J. Collimation of sound assisted by acoustic surface waves. Nat. Phys. 3, 851-852 (2007).

29 Sievenpiper, D., Zhang, L., Broas, R. F., Alexopolous, N. G. \& Yablonovitch, E. Highimpedance electromagnetic surfaces with a forbidden frequency band. IEEE Trans. Microw. Theory Tech. 47, 2059-2074 (1999).

30 Kosaka, H., Kawashima, T., Tomita, A., Notomi, M., Tamamura, T., Sato, T. \& Kawakami, S. Self-collimating phenomena in photonic crystals. App. Phys. Lett. 74, 1212 (1999).

31 Gao, W. L., Fang, F. Z., Liu, Y. M. \& Zhang, S. Chiral surface waves supported by biaxial hyperbolic metamaterials. Light Sci. Appl. 4, e328 (2015).

32 Luo, C., Johnson, S. G., Joannopoulos, J. D. \& Pendry, J. B. All-angle negative refraction without negative effective index. Phys. Rev. B 65, 201104 (2002).

33 Bliokh, K. Y., Smirnova, D. \& Nori, F. Quantum spin Hall effect of light. Science $\mathbf{3 4 8}$ 1448-1451 (2015).

34 Lin, J., Mueller, J. B., Wang, Q., Yuan, G., Antoniou, N., Yuan, X.-C. \& Capasso, F. Polarization-controlled tunable directional coupling of surface plasmon polaritons. Science 340, 331-334 (2013).

35 Rodríguez-Fortuño, F. J., Marino, G., Ginzburg, P., O'Connor, D., Martínez, A., Wurtz, G. A. \& Zayats, A. V. Near-field interference for the unidirectional excitation of electromagnetic guided modes. Science 340, 328-330 (2013).

36 Petersen, J., Volz, J. \& Rauschenbeutel, A. Chiral nanophotonic waveguide interface based on spin-orbit interaction of light. Science 346, 67-71 (2014).

37 Xu, Y., Zhang, X., Tian, Z., Gu, J., Ouyang, C., Li, Y., Han, J. \& Zhang, W. Mapping the near-field propagation of surface plasmons on terahertz metasurfaces. App. Phys. Lett. 107, 021105 (2015).

38 Zhang, X., Xu, Y., Yue, W., Tian, Z., Gu, J., Li, Y., Singh, R., Zhang, S., Han, J. \& Zhang, W. Anomalous surface wave launching by handedness phase control. Adv. Mater. 27, 7123-7129 (2015).

This work is licensed under a Creative Commons Attribution 4.0 International License. The images or other third party material in this article are included in the article's Creative Commons license, unless indicated otherwise in the credit line; if the material is not included under the Creative Commons license, users will need to obtain permission from the license holder to reproduce the material. To view a copy of this license, visit http:// creativecommons.org/licenses/by/4.0/

(C) The Author(s) 2017 